\title{
Os Efeitos da Doxorrubicina na Biossíntese e no Metabolismo do Heme em Cardiomiócitos
}

\author{
Effects of Doxorubicin on Heme Biosynthesis and Metabolism in Cardiomyocyte \\ Zuoyan Wang, ${ }^{1}$ Junyi Gao, ${ }^{1}$ Haobo Teng, ${ }^{1}$ Jianjun Peng ${ }^{10}$ \\ Capital Medical University Affiliated Beijing Shijitan Hospital, ${ }^{1}$ Beijing - China
}

\section{Resumo}

Fundamento: A doxorrubicina está associada à cardiotoxicidade e à morbidade cardíaca tardia. O heme está relacionado ao stress oxidativo celular. Entretanto, sua regulação específica em cardiomiócitos sob os efeitos de doxorrubicina ainda não foi documentada.

Objetivo: Nosso objetivo é avaliar as alterações de enzimas limitantes de velocidade no caminho metabólico do heme sob o efeito de doxorrubicina.

Métodos: Cardiomiócitos H9c2 com doxorrubicina em concentrações diferentes $(1,2,5,10 \mu \mathrm{M}$ respectivamente). Os testes de PCR em tempo real e Western Blot foram usados para determinar a expressão de proteína e mRNA para quatro enzimas cruciais (ALAS1, ALAS2, HOX-1, e HOX-2) que regulam o metabolismo do heme celular, e os níveis de heme foram detectados por ELISA. Um $p<0,01$ foi considerado significativo.

Resultados: Observamos um padrão com alteração dependendo da dose nos níveis de heme nas células H9c2 com o nível mais alto na concentração de $5 \mu \mathrm{M}$ de doxorrubicina, o que ocorreu sincronicamente com o nível mais alto de regulação para cima de ALAS1, bem como as enzimas degenerativas HOX-1 e HOX-2 na expressão de proteína e mRNA. Em contraste, observamos que a ALAS2 foi regulada para baixo gradualmente, inversamente proporcional às concentrações de doxorrubicina.

Conclusão: O aumento da expressão de ALAS1 pode ter um papel na elevação do nível do heme quando o cardiomiócito H9c2 for exposto à doxorrubicina, e pode ser um alvo terapêutico para a toxicidade miocárdica induzida por doxorrubicina. (Arq Bras Cardiol. 2021; 116(2):315-322)

Palavras-chave: Doxorrubicina; Biosíntese; Heme; Miócitos Cardíacos; Cardiotoxicidade; ALAS.

\begin{abstract}
Background: Doxorubicin is associated with cardiotoxicity and late cardiac morbidity. Heme is related to cellular oxidative stress. However, its specific regulation in cardiomyocytes under doxorubicin effects has not yet been documented.

Objective: This study seeks to evaluate the changing profiles of rate-limiting enzymes in the heme metabolism pathway under the effect of doxorubicin.

Methods: H9c2 cardiomyocytes were incubated with doxorubicin at different concentrations (1,2,5,10 $\mathrm{MM}$ respectively). The real-time PCR and Western Blot were used to determine the mRNA and protein expression for four pivotal enzymes (ALAS1, ALAS2, HOX-1, and HOX-2) regulating cellular heme metabolism, as well as the levels of heme were detected by ELISA. $p<0.01$ was considered significant.

Results: This study observed a dose-dependent changing pattern in heme levels in H9c2 cells with the highest level at the 5 $\mu \mathrm{M}$ concentration for doxorubicin, which occurred synchronously with the highest upregulation level of ALAS1, as well as the degradative enzymes, HOX-1, and HOX-2 in mRNA and protein expression. By contrast, ALAS2, contrary to the increasing concentrations of doxorubicin, was found to be progressively down-regulated.

Conclusion: The increase in ALAS1 expression may play a potential role in the heme level elevation when H9c2 cardiomyocyte was exposed to doxorubicin and may be a potential therapeutic target for doxorubicin-induced myocardial toxicity. (Arq Bras Cardiol. 2021; 116(2):315-322)
\end{abstract}

Keywords: Doxorubicin; Biosynthesis; Heme; Myocites, Cardiac; Cardiotoxicity; ALAS.

Full texts in English - http://www.arquivosonline.com.br

Correspondência: Jianjun Peng •

Capital Medical University Affiliated Beijing Shijitan Hospital - No.10 Tieyi Road, Haidian District, Beijing, 100038, China Beijing 100038

E-mail: pengjianjun2361@bjsjth.cn

Artigo recebido em 30/06/2019, revisado em 19/11/2019, aceito em 27/12/2019

DOI: https://doi.org/10.36660/abc.20190437 


\section{Introdução}

Com a progressão contínua das drogas antitumorais e radioterapia, o índice de sobrevivência dos pacientes com tumores malignos melhorou, e seu período de sobrevivência é significativamente aumentado. Entretanto, o uso disseminado de drogas antitumorais é acompanhado de um aumento dos eventos cardiovasculares adversos, que afeta a sobrevivência e a qualidade de vida do paciente. Antraciclinas são drogas derivadas da estreptomicina, incluindo a doxorrubicina, e da epirrubicina, que são amplamente utilizadas para tratar câncer de mama, câncer do pulmão de pequenas células, mieloma, sarcoma, linfoma e leucemia. A miocardiopatia e a insuficiência cardíaca subsequente são as manifestações mais graves de cardiotoxicidade causadas por drogas antraciclinas na quimioterapia. Além disso, a toxicidade cardiovascular das antraciclinas depende da dose e é irreversível. ${ }^{1,2}$

O mecanismo exato da toxicidade miocárdica induzida por drogas antraciclinas ainda não é clara, embora várias teorias tenham sido propostas, incluindo a inibição da replicação de DNA e transcrição de RNA; o dano ao DNA causado por radicais livres, peroxidação lipídica e alquilação; a ligação cruzada do DNA; interferência no desenrolamento do DNA; a inibição da topoisomerase II, etc.

O heme é uma porfirina ligante importante, como suplemento da proteína do heme, tem a função fisiológica de transportar o $\mathrm{O}_{2}$ no corpo. Muitas funções biológicas relacionadas à vida, tais como o transporte de elétrons, o armazenamento de oxigênio, a transdução de sinal, e a expressão genética são controladas pelas várias proteínas heme. Estudos recentes detectaram que os níveis de heme aumentaram significativamente em modelos de insuficiência cardíaca isquêmica miocárdica de ratos, sugerindo que o heme possa ter um papel importante em lesões miocárdicas isquêmicas e hipóxicas. ${ }^{3,4}$ Entretanto, não há trabalhos publicados na literatura que exploram a alteração da biossíntese ou do metabolismo do heme em cardiomiócitos, sob tratamento com antraciclinas.

A biossíntese do heme é iniciada pela formação do ácido ס-aminolevulínico (ALA) a partir da glicina (Gly) e succinil coenzima A, catalisada pela sintase do ALA (ALAS), que tem duas isoenzimas: ALAS1 e ALAS2. Por outro lado, a heme oxigenase (HOX) media o primeiro passo do catabolismo do heme, e divide o heme para formar a biliverdina. Duas isoformas ativas de HOX foram identificadas, a HOX-1 induzível, e a menos regulada HOX-2.

O objetivo deste estudo foi elucidar as alterações de perfis de quatro enzimas cruciais, a sintase do ácido aminolevulínico 1 (ALAS1), a sintase do ácido aminolevulínico 2 (ALAS2), heme oxigenase 1 (HOX-1), e heme oxigenase 2 (HOX-2), no cardiomiócito H9c2 em tratamento com doxorrubicina.

\section{Métodos}

\section{Cultura celular e tratamento com doxorrubicina}

Foram comprados cardiomiócitos H9c2 da American Type Culture Collection (ATCC, Manassas, VA, EUA) e foi feita a cultura em meio DMEM/F12 contendo 10\% de soro fetal bovino e $1 \%$ de meio de anticorpo duplo, nas condições de $37^{\circ} \mathrm{C}$ e
5\% de CO2, em câmara umidificada. As células de H9c2 foram distribuídas em placas de titulação de 6 poços, num índice de $2 \times 10^{5}$ células/poço em um volume de $2 \mathrm{~mL}$ e em cultura de meio DMEM/F12 contendo 10\% de soro fetal bovino, por 24 h. Depois disso, doses diferentes de doxorrubicina $(1,2,5,10$ $\mu \mathrm{M}$, respectivamente) foram adicionados aos poços da placa de titulação. Consideramos células não tratadas com doxorrubicina (tratadas com solução salina) como grupo de controle. Depois de 24 horas de incubação a $37^{\circ} \mathrm{C}$, as células de poços diferentes foram coletadas separadamente por centrifugação (10000 rpm por 10 minutos a $4^{\circ} \mathrm{C}$ ) e usadas para estudos posteriores abaixo.

\section{PCR quantitativo em tempo real}

O RNA total foi extraído das células $\mathrm{H} 9 \mathrm{c} 2$ de todos os grupos utilizando o TRIzol (Invitrogen; Thermo Fisher Scientific, Inc., Waltham, MA, EUA), e foi feita a transcrição reversa com o kit SuperScript Double-Strand Synthesis (Invitrogen; Thermo Fisher Scientific, Inc., Waltham, MA, USA) de acordo com as instruções do fabricante, e amplificado em um sistema de PCR de tempo real 7500 Fast com SYBR GreenER qPCR SuperMix Universal (Invitrogen; Thermo Fisher Scientific, Inc.). Os primers usados para amplificar ALAS1, ALAS2, HOX-1, e HOX-2 foram projetados usando os softwares Primer3 (v. 0.4.0). Os seguintes primers foram usados para o PCR em tempo real:

\section{ALAS1-F:TTGCCAAAGTCCGTTTCC}

R: TGTAGTCATCTGCCATAGGG 3';

\section{ALAS2-F: TCAAGGGAGAGGAGGGTCAAG \\ R: ACGAGGCACAGTTGGGTAG \\ HOX-1-F:TCGACAACCCCACCAAGTT \\ R:CTGGCGAAGAAACTCTGTCT \\ HOX-2-F:GCTTACACTCGTTACATGGG \\ R: CACATGCTCGAACAGGTAGA \\ GAPDH-F: GATGACATCAAGAAGGTGGTGA \\ R:ACCСTGTTGCTGTAGCCATATTC.}

As condições de reação foram as seguintes: $95^{\circ} \mathrm{C}$ por 3 min; $95{ }^{\circ} \mathrm{C}$ por $30 \mathrm{seg}, 55^{\circ} \mathrm{C}$ por $20 \mathrm{seg}, 72{ }^{\circ} \mathrm{C}$ por 20 seg com 40 ciclos. Foram realizadas análises de curva de fusão para verificar a especificidade de sua amplificação. Os valores de expressão de todos os genes-alvo de cada amostra foram calculados normalizando com GAPDH controle interno, e calculados utilizando o método $2-\triangle \triangle C T$.

\section{Western Blot}

Células H9c2 foram centrifugadas por tripsinização por 5 min a 1.000 x g e lavadas com PBS frio. Em seguida, as células $\mathrm{H} 9 \mathrm{c} 2$ foram ressuspensas em tampão de lise com inibidores de protease e fosfatase. O lisado celular foi mantido em gelo e agitado no vórtex. Depois da centrifugação por 20 a $13,000 \times \mathrm{g}$, o sobrenadante foi separado e armazenado a $-80{ }^{\circ} \mathrm{C}$ até o uso. Depois da desnaturação, $20 \mu \mathrm{g}$ de proteína total foram colocados em eletroforese em gel de acrilamida contendo dodecil sulfato de sódio 12\% (SDS-PAGE), e foram transferidos por eletroforese para uma membrana de fluoreto de polivinilideno (PVDF). Depois do bloqueio com TBST (50mM Tris- $\mathrm{HCl}, \mathrm{pH} 8 ; 154 \mathrm{mM} \mathrm{NaCl}$ e $0,1 \%$ tween20) por 2 horas em temperatura ambiente, as membranas foram 
testadas com anticorpos específicos em relação a ALAS1 (diluição,1:1.000; policlonal de coelho; no cat. 16200-1-AP; Proteintech), ALAS2 (diluição, 1:1.000; policlonal de coelho; $\mathrm{n}$ 은 cat. ab136799; Abcam), HOX-1 (diluição,1:1.000; policlonal de coelho; no cat. ab230513; Abcam), HOX-2(diluição, 1:500; policlonal de coelho; no cat. ab229960; Abcam) e GAPDH (diluição, 1:1.000; clone 6C5; cat. no. ab8245; Abcam) durante a noite a $4^{\circ} \mathrm{C}$. Anticorpos secundários titulados com Horseradish peroxidase (HRP) (1:1.000 diluição; no cat. A0208, A0216; Beyotime) foram acrescentados, após a incubação em temperatura ambiente por 2 horas. As membranas foram visualizadas com kits de quimioluminescência amplificada (Biorbyt, Ltd., Cambridge, Reino Unido). Para quantificar a expressão de proteína direcionada, os filmes de raio $\mathrm{X}$ foram escaneados, e as intensidades de banda foram quantificadas pelo software ImageJ 1.47 . Os resultados foram normalizados em GAPDH.

\section{Medição de heme intracelular}

O ensaio de imunoabsorção enzimática (ELISA) foi utilizado para determinar os níveis de heme intracelular. As células H9c2 de cada grupo foram lisadas e submetidas a centrifugação a 12000rpm por 15 minutos a $4{ }^{\circ} \mathrm{C}$ para retirar detritos. A concentração de proteína do lisado celular foi quantificada pelo kit de Ensaio de Proteína BCA (Beyotime). O heme nos lisados celulares de H9c2 foi medido utilizando-se o Ensaio Heme QuantiChrom (BioAssay Systems), seguindo o protocolo do fabricante e normalizado a uma concentração de proteína de cada amostra.

Citometria de fluxo para determinar o índice de apoptose em células H9c2

A apoptose foi avaliada utilizando-se o kit Annexin V-FITC/ PI Apoptosis Detection kit (KeyGEN, China) de acordo com o protocolo do fabricante. Depois da intervenção, aproximadamente $1 \times 10^{5}$ células $\mathrm{H} 9 \mathrm{c} 2$ de cada grupo foram lavadas, digeridas com tripsina e as células ressuspensas com $1 \times$ PBS $\left(4^{\circ} \mathrm{C}\right)$, centrifugadas a $2.000 \mathrm{rpm}$ por 5-10 minutos para lavar as células. As células foram ressuspensas em $500 \mu \mathrm{l}$ do tampão, seguido da adição de $5 \mu \mathrm{l}$ de Annexin V-FITC e $5 \mu \mathrm{l}$ de Pl. As células foram incubadas no escuro por 15 minutos em temperatura ambiente. Em seguida, as células de cada grupo foram examinadas quanto ao índice de apoptose por citometria de fluxo (BD Accuri ${ }^{\mathrm{TM}}$ C6), e o experimento foi repetido três vezes. $\mathrm{O}$ índice de apoptose foi quantificado como a porcentagem de células coradas com Annexin V.

\section{Análise estatística}

Os dados no presente estudo são normalmente distribuídos, o que foi verificado pelo teste de Shapiro-Wilk, e são expressos como média \pm SD. A ANOVA de fator único, seguida do teste post hoc de Tukey, foi usada para examinar a significância estatística das diferenças entre os grupos. A análise estatística foi realizada utilizando-se o software SPSS 24.0 Statistical Package Program for Windows (SPSS Inc., Chicago, IL, EUA). Um p-valor bilateral de $<0,01$ foi considerado estatisticamente significativo.

\section{Resultados}

\section{Alterações nos níveis de heme em células H9c2 com concentrações diferentes de doxorrubicina}

Conforme mostrado na Figura 1, em comparação com o grupo de controle $(5088,4 \pm 153,1 \mathrm{ng} / \mathrm{ml})$, os níveis de heme das células $\mathrm{H} 9 \mathrm{c} 2$ foram significativamente regulados para cima 1,27 vezes $(6493,1 \pm 138,8 \mathrm{ng} / \mathrm{ml}), 1,56$ vezes $(7498,9 \pm 110,2 \mathrm{ng} / \mathrm{ml})$ e no máximo um aumento de 2,34 vezes $(11896,6 \pm 187,3 \mathrm{ng} / \mathrm{ml})$

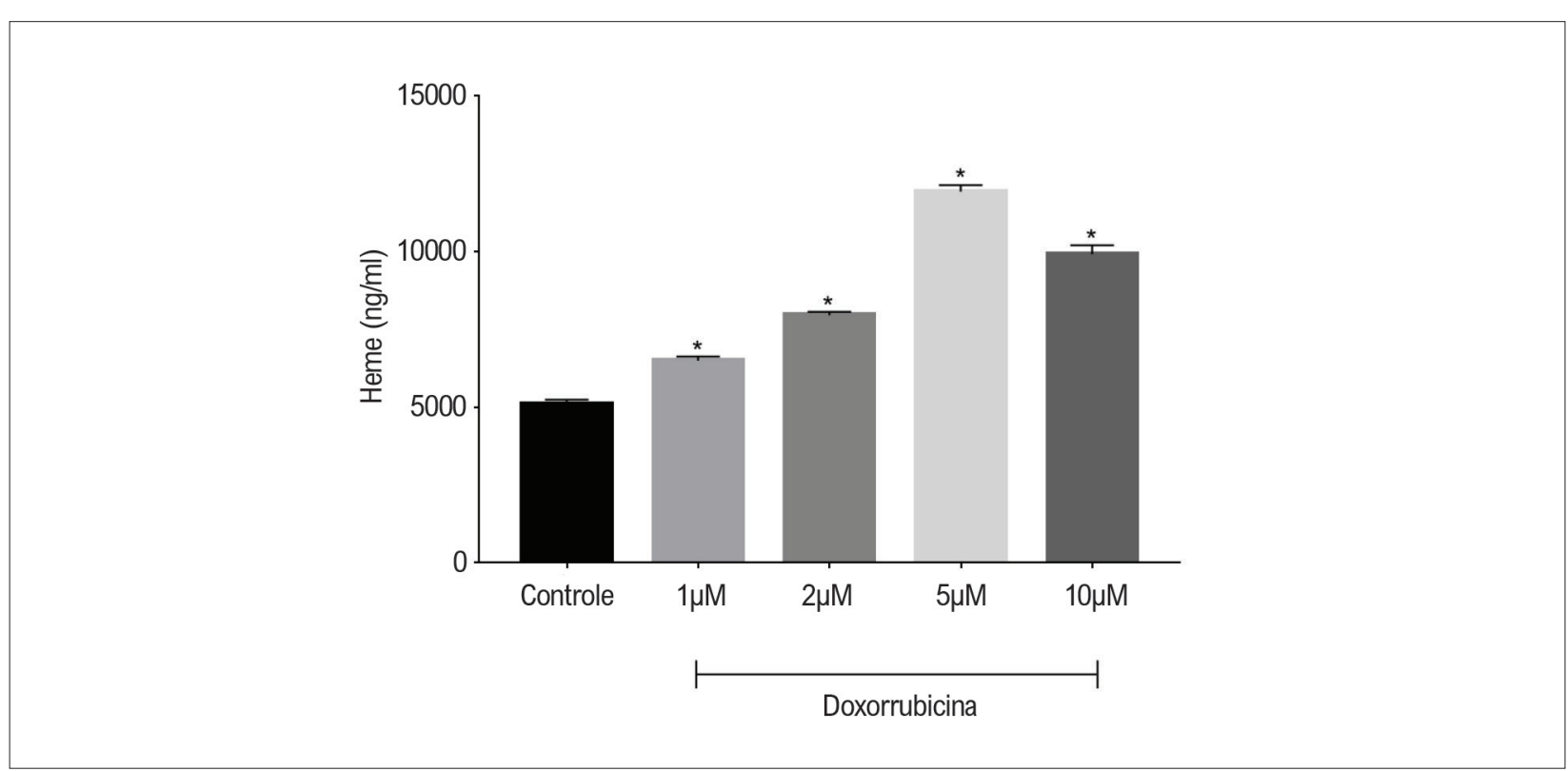

Figura 1 - Efeitos da doxorrubicina nos niveis de heme nas células H9c2 exposta a solução salina (grupo de controle) ou doxorrubicina com concentração diferente por 24 horas. Niveis de heme medidos por ELISA. Os dados são apresentados como média \pm desvio padrão. * $p<0,01$, em comparação ao grupo de controle. 
nos grupos com $1 \mu \mathrm{M}, 2 \mu \mathrm{M}$, e $5 \mu \mathrm{M}$ de doxorrubicina, separadamente $(p<0,01)$. Essa tendência à regulação para cima passou a ter um aumento de 1,95 vezes $(9911,9 \pm 286,8 \mathrm{ng} / \mathrm{ml})$ no grupo com $10 \mu \mathrm{M}$ de doxorrubicina, em comparação com grupo de controle $(p<0,01)$.

Efeitos da doxorrubicina nos índices de apoptose de células H9c2

A análise de citometria de fluxo demonstrou que, em comparação com o grupo de controle tratado com solução salina, o índice de apoptose de células $\mathrm{H} 9 \mathrm{c} 2$ tratadas com concentrações diferentes de doxorrubicina aumentou significativamente, conforme mostrado na Figura 2. Quando incubado com 1,2,5, e 10 $\mu \mathrm{M}$ de doxorrubicina por 24 horas, o índice total de apoptose, incluindo a apoptose de estágio inicial e final, de H9c2 foi aumentado para 10,6 $\pm 1,6 \%$, $41,1 \pm 1,9 \%, 60,5 \pm 3,6 \%$, e $76,0 \pm 2,5 \%$ respectivamente, em comparação com $2,1 \pm 0,5 \%$ no grupo de controle $(p<0,01)$.

Regulação da sintase do ácido aminolevulínico 1 (ALAS1) e da sintase do ácido aminolevulínico 2 (ALAS2) expressão de RNA mensageiro ( $m$ RNA) em células H9c2 depois do tratamento com doxorrubicina

Como primeiro passo, foram avaliadas as enzimas limitantes de velocidade da síntese do heme que ocorre nas mitocôndrias, a expressão do mRNA da ALAS1 e ALAS2 em tratamento com doxorrubicina. Depois da incubação com $1 \mu \mathrm{M}$ e $2 \mu \mathrm{M}$ doxorrubicina, as expressões de mRNA no ALAS1 foram reguladas para baixo (embora não sejam estatisticamente significativos). Depois de tratar com $5 \mu \mathrm{M}$ e $10 \mu \mathrm{M}$ de doxorrubicina, as expressões de mRNA de ALAS1 foram estatisticamente aumentadas até 41,1 vezes 375,3 vezes, em comparação com o grupo de controle separadamente. Diferente da ALAS1, a expressão de mRNA de ALAS2 demonstrou um uma regulação para baixo significativa (grupo $1 \mu \mathrm{M}: 0,88$ vezes, grupo $2 \mu \mathrm{M}$ : 0,83vezes, grupo de $5 \mu \mathrm{M}: 0,49$ vezes, grupo $10 \mu \mathrm{M}: 0,31$ vezes respectivamente, conforme mostrado na Figura 3A.)

Regulação da expressão do mRNA para as HOX-1 e HOX-2 em células H9c2 após o tratamento com doxorrubicina

Foram examinadas as enzimas citoplasmáticas limitantes de velocidade in catabolismo do heme, HOX-1, e HOX2. Detectamos que, depois de incubado com um nível crescente de doxorrubicina, o mRNA de HOX-1 e HOX-2 exibiu o mesmo padrão de regulação, embora com níveis de alteração diferenciados. Não houve alteração significativa na expressão do mRNA depois do tratamento com $1 \mu \mathrm{M}$ e $2 \mu \mathrm{M}$, comparado com o grupo de controle, nem em HOX-1, nem HOX-2. Depois de incubado com $5 \mu \mathrm{M}$ de doxorrubicina, a expressão de mRNA para HOX-1 e HOX-2 se regularam para uma mudança para cima de 4,3 vezes e 15,5 vezes, respectivamente $(p<0,01)$. Entretanto, após o tratamento com $10 \mu \mathrm{M}$ de doxorrubicina, a regulação para cima diminuiu até o nível de 2,6 vezes e 3,2 vezes separadamente, em comparação com o grupo de controle, $(p<0,01)$, conforme mostrado na Figura 3B

Regulação dos níveis de proteína de ALAS1, ALAS2, HOX-

\section{1, e HOX-2 após o tratamento com doxorrubicina}

A análise de Western Blot demonstrou que, de forma idêntica ao padrão de expressão de mRNA, os níveis de proteína ALAS1 foram regulados para baixo significativamente no grupo de $2 \mu \mathrm{M}$ de doxorrubicina, e regulado para cima no grupo de $10 \mu \mathrm{M}$ de doxorrubicina, em comparação com o grupo de controle. Observou-se que os níveis de proteína
A

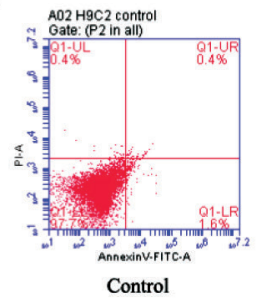

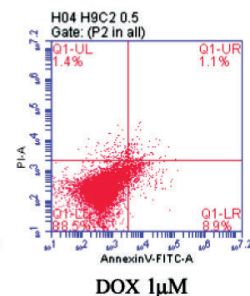
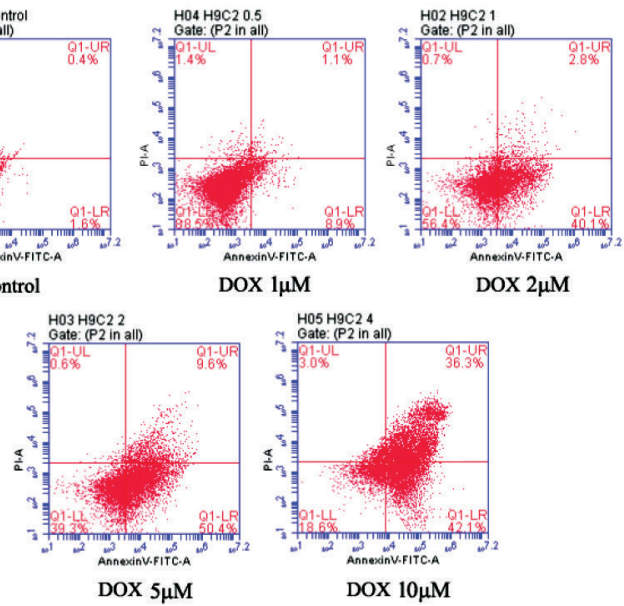

B

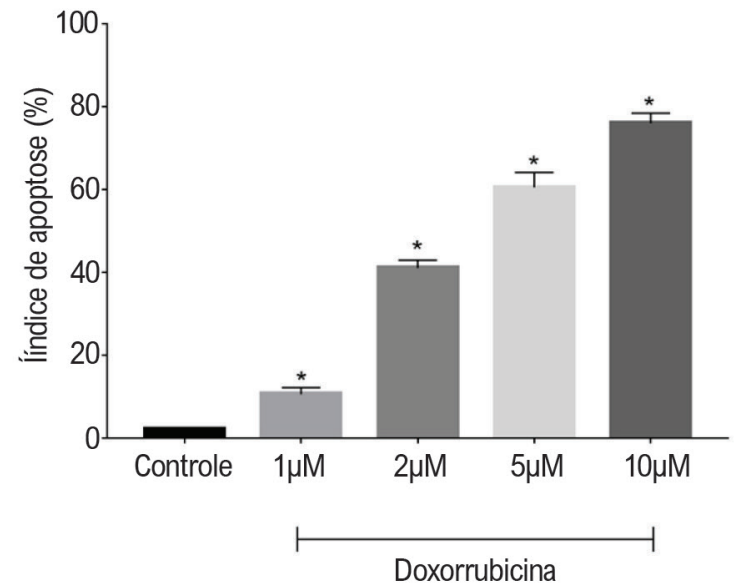

Figura 2-Análise por citometria de fluxo dos efeitos da doxorrubicina na viabilidade das células H9c2 As células H9c2 foram pré-tratadas com solução salina (controle) e doxorrubicina a 1,2,5 e 10 $\mathrm{M}$ respectivamente por 24h. (A) Análises representativas de citometria de fluxo de cinco experimentos separados correspondentes ao controle a às várias concentrações de tratamento com doxorrubicina, respectivamente. (B) Gráfico estatístico de coloração annexin V-FITC/PI. Resultados expressos como média \pm desvio padrão. * ${ }^{*}<0,01$, em comparação ao grupo de controle. 


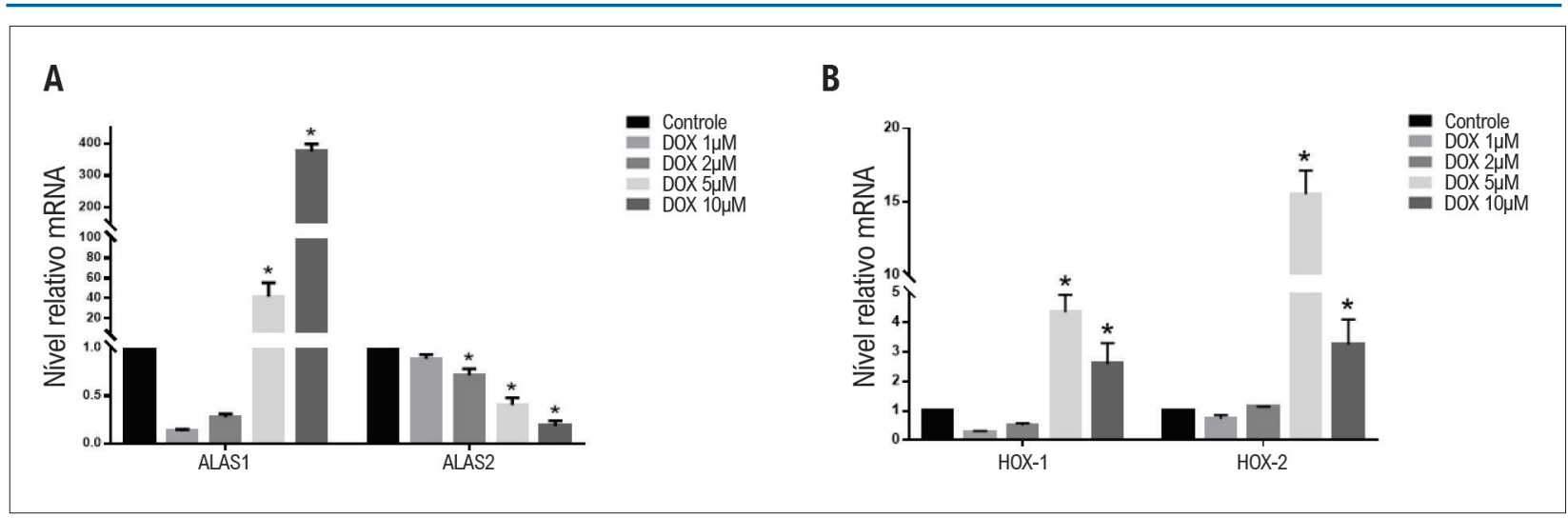

Figura 3 - (A) alteração da expressão de mRNA de ALAS1 e ALAS2 em tratamento com doxorrubicina. sintase do ácido aminolevulínico 1; ALAS2, sintase do ácido aminolevulínico 2. (B) Características da alteração da expressão de mRNA para enzimas catabólicas do heme em tratamento com doxorrubicina. HOX-1, heme oxigenase 1 ; HOX-2, heme oxigenase2. * indica $p<0,01$ versus grupo de controle.

foram regulados para baixo progressivamente, à medida que a concentração de doxorrubicina aumentou de $1 \mu \mathrm{M}$ a $10 \mu \mathrm{M}$, conforme mostrado na Figura 4A.

A expressão de proteína de HOX-1 foi ligeiramente regulada para baixo no tratamento com $1 \mu \mathrm{M}$ e $2 \mu \mathrm{M}$ de doxorrubicina, e depois regulada para cima de forma abrupta, em comparação com o grupo de controle. No entanto, de forma inconsistente com o padrão de expressão de mRNA, detectamos um aumento progressivo no nível de proteína HOX-2 com o aumento da concentração de doxorrubicina de $1 \mu \mathrm{M}$ a $5 \mu \mathrm{M}$, e, em seguida, voltou para o nível de linha de base quando tratado com $10 \mu \mathrm{M}$ de doxorrubicina (Figura 4B).

\section{Discussão}

Doxorrubicina é um tipo de droga antraciclina que é uma droga antitumoral eficiente de amplo espectro. Ela é amplamente utilizada no tratamento de vários tumores malignos, tais como câncer de mama, câncer de pulmão e linfoma. ${ }^{5}$ Entretanto, os efeitos cardíacos tóxicos da doxorrubicina na dose clínica para quimioterapia são graves e dependem da dose, o que pode levar a cardiomiopatia e insuficiência cardíaca congestiva, e, portanto, limitam muito seu uso clínico. ${ }^{6}$ Diferente do mecanismo de sua atividade antitumoral, o mecanismo primário de toxicidade cardíaca induzido pela doxorrubicina, é a geração de espécies reativas do oxigênio (ERO) e a promoção subsequente do stress oxidativo miocárdico. ${ }^{7} \mathrm{O}$ heme é um mediador essencial da disponibilidade bioquímica do ferro. ${ }^{8}$ A função da molécula do heme varia de acordo com a proteína ligante com a qual é coordenado. Ele pode funcionar como mediador de transporte e armazenamento de oxigênio em hemoglobina ${ }^{9}$ ou mioglobina, ${ }^{10} \mathrm{e}$, por outro lado, ele age como um transportador de elétrons em citocromos, e é a fonte crítica de ferro redox-ativo. ${ }^{11}$ Bhoite-Solomon et al. identificaram que o heme livre é tóxico para o miocárdio, e causa citólise por meio de dano no sarcolema, dependendo da concentração. ${ }^{12}$ Além dos cardiomiócitos, o heme livre também pode ser tóxico às células epiteliais humanas e a células semelhantes a neurônios, ${ }^{13,14}$ por meio do stress oxidativo causado pela apoptose ou necrose da célula. Além disso, o heme livre pode causar dano celular endotelial, ao estimular a expressão de fatores inflamatórios. ${ }^{15,16}$

Entretanto, diferentemente dos vários estudos sobre dano oxidativo a cardiomiócitos mediados por ferro tratados com drogas antraciclinas, a variação do nível de heme intracelular e a regulação dos processos de sua síntese e metabolismo não foram bem avaliados no cardiomiócito em tratamento com antraciclina. No presente estudo, examinamos sistematicamente as características de variação de enzimas biossintéticas e degradadoras do heme pela primeira vez.

No caminho de biossíntese, há oito enzimas envolvidas, dentre as quais quatro são enzimas mitocondriais, e quatro são enzimas citoplasmáticas. ${ }^{17} \mathrm{Na}$ primeira etapa da síntese do heme, a glicina e succinil coenzima A são condensadas no ácido ठ-aminolevulínico (ALA). Essa reação precisa ser catalisada pela sintase do ácido aminolevulínico (ALAS). ${ }^{18}$ Há dois tipos de ALAS: ALAS1 e ALAS2. A primeira tem uma expressão universal, enquanto a última é predominantemente expressa em células precursoras de hemácias. ${ }^{19}$ No presente estudo, observamos que não apenas a ALAS1, mas também a ALAS2 foram expressas nas células $\mathrm{H} 9 \mathrm{c} 2$ em níveis de linha de base. Após o tratamento com doxorrubicina, a expressão de mRNA de ALAS1 e ALAS2 apresentam dois padrões de alteração distintos. A expressão de proteína e mRNA de ALAS1, a princípio, apresentou uma tendência à inibição (embora não fosse estatisticamente significativa em nosso estudo) com $1 \mu \mathrm{M}$ e $2 \mu \mathrm{M}$ de doxorrubicina, e, depois, padrões dramáticos de regulação para cima com o tratamento com $5 \mu \mathrm{M}$ e $10 \mu \mathrm{M}$ de doxorrubicina.

Por outro lado, a expressão de proteína e mRNA de ALAS2 foi suprimida gradativamente até o nível mais baixo, quando tratada com $10 \mu \mathrm{M}$ de doxorrubicina, com $69,0 \%$ e $35,0 \%$ de redução, respectivamente. Esse fenômeno indica que, sob o efeito da doxorrubicina, a regulação de ALAS1 e ALAS2 pode tomar caminhos diferentes e ter objetivos de bioprocessos direcionadores diferentes. A regulação para baixo de ALAS2 pode ser explicada pelo feedback negativo de uma elevação progressiva do nível de heme. Esse efeito pode ser alcançado reprimindo-se a transcrição de mRNA de ALAS2, conforme mostrado em nossos resultados de PCR em tempo real, bem como interrompendo-se a formação de 


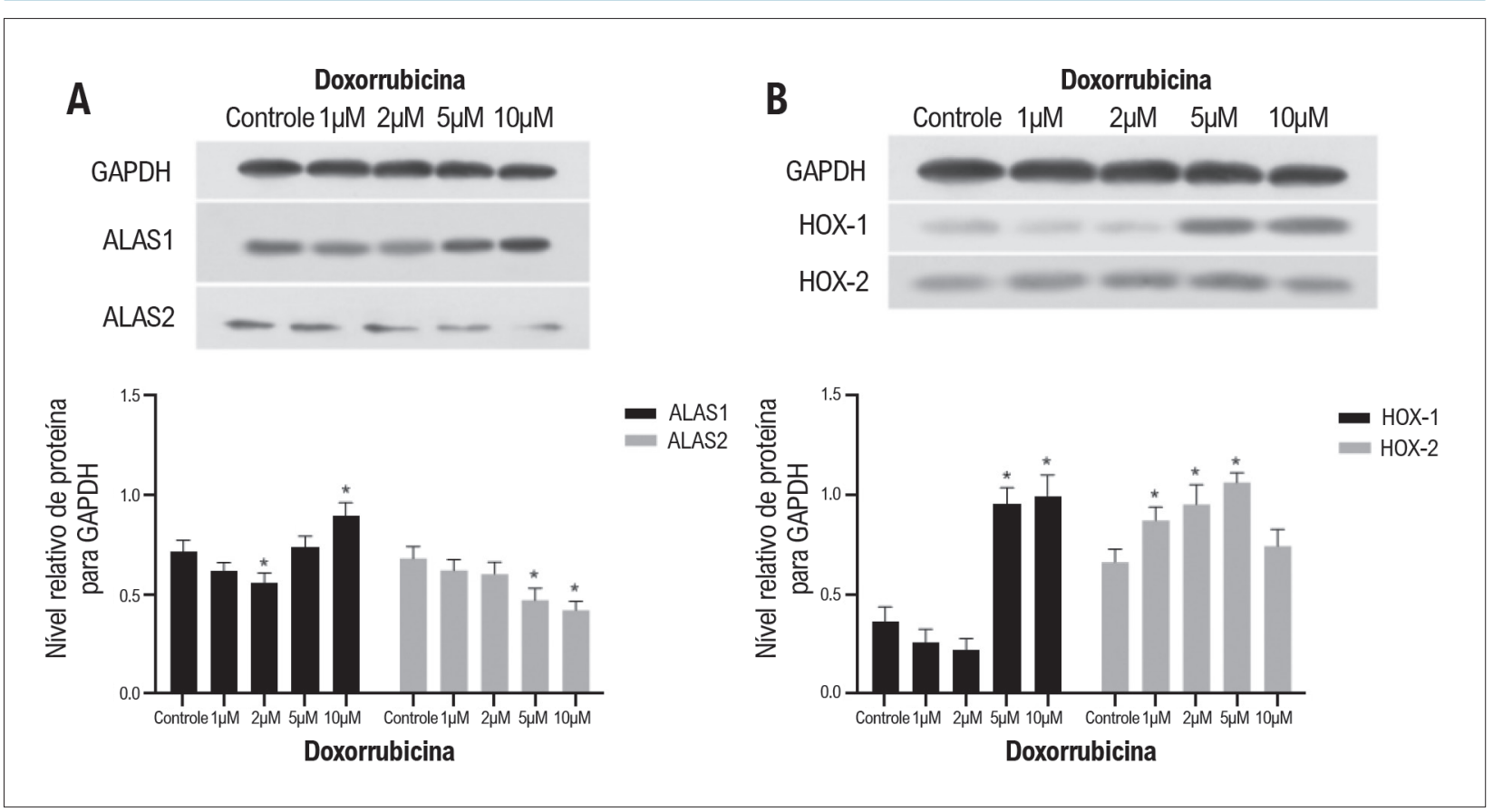

Figura 4 - Análise de dados de Western Blot para alterações de níveis de proteína ALAS (A) e HOX (B) em células H9c2 em concentrações de doxorrubicina diferentes. ALAS1: sintase do ácido aminolevulínico 1; ALAS2: sintase do ácido aminolevulínico; HOX-1: heme oxigenase 1; HOX-2: heme oxigenase 2. Cada teste Western Blot foi realizado três vezes. * indica $p<0,01$ versus grupo de controle.

ALAS2 por meio de um bloco do precursor de ALAS2 até as mitocôndrias. ${ }^{20,21}$ Nas hemácias, a expressão de ALAS2 é determinada pela transativação de locais de fatores nucleares GATA-1, CACC box, e NF-E2-ligante na área promotora, e sua síntese é regulada pela quantidade de ferro livre. ${ }^{22}$ Entretanto, os mecanismos de regulação de ALAS2 em cardiomiócitos em tratamento com doxorrubicina precisa ser pesquisada mais a fundo.

Em nosso estudo, ALAS1, que deveria estar estritamente em controle de feedback negativo em nível baixo para evitar o alto nível citotóxico de heme, foi expresso excessivamente nos aspectos do gene e da proteína, apesar do alto nível de heme que existe simultaneamente. Podvinec et al., ${ }^{23}$ relataram que as drogas que induzem citocromos P450 e outras enzimas associadas que metabolizam drogas podem fazer a regulação para cima de ALAS1 simultaneamente e por meio de transcrição, que foi o primeiro passo na síntese de heme, para coordenar com a necessidade de atividade de citocromo P450 com regulação para cima. Esse processo foi mediado por dois elementos aperfeiçoadores, localizados a 20 e $16 \mathrm{~kb}$ a montante do local de início transcricional de ALAS1, e sua interação com os xeno-receptores NR1I2 e NR1I3. ${ }^{23}$ Zordoky et al., ${ }^{24}$ revelaram que a doxorrubicina causa indução significativa de vários genes $\mathrm{P} 450$ do citocromo, tais como expressão genética de CYP1A1, CYP1A2, CYP1B1, e CYP2B2 dependendo da concentração. NR1I2 e NR1I3 também demonstraram ser os receptores nucleares cruciais que mediaram a interação entre a doxorrubicina e as enzimas metabolizantes. ${ }^{25}$ Os mecanismos precisos de regulação de padrões de expressão de ALAS no cardiomiócito precisará ser elucidado em estudos futuros.
A heme oxidase (HOX) é a enzima limitante de velocidade no processo de degradação do heme, que pode degradar por oxidação a molécula do heme para produzir monóxido de carbono, ferro e biliverdina. A heme oxidase tem duas isoenzimas, heme oxidase 1 ( $\mathrm{HOX}-1)$ e heme oxidase 2 ( $\mathrm{HOX}$ 2). A HOX-2 desempenha basicamente um papel regulatório sob condições fisiológicas normais, enquanto a HOX-2 pode proteger as células e os tecidos em stress oxidativo. ${ }^{26}$

A expressão de HOX-1 pode ser promovida por uma variedade de estímulos, incluindo a doxorrubicina ${ }^{27}$ e o acúmulo de heme intracelular. Estudos anteriores revelaram que a expressão de HO-1 é regulada principalmente no nível da transcrição, e identificou-se que vários elementos regulatórios têm um papel importante na regulação para cima da HOX-1, incluindo elementos de resposta metal (MREs), elementos de resposta ao stress (StREs), AP-1, e NF-B. ${ }^{28}$ Por outro lado, demonstrou-se que Bach1 tem um efeito repressor na expressão de HOX-1. ${ }^{29}$ No presente estudo, identificamos que a HOX-1 nos cardiomiócitos de H9c2 foi inicialmente regulada para baixo quando tratada com baixa concentração de doxorrubicina ( $1 \mu \mathrm{M}$ e $2 \mu \mathrm{M})$ nas células H9c2 e, em seguida significativamente regulada para cima com incubação com $5 \mu \mathrm{M}$ e $10 \mu \mathrm{M}$ de doxorrubicina. Nossa pesquisa indica que serão necessários mais estudos com foco na interação entre vários reguladores de e elementos de gene da HOX-1 em concentrações diferentes de doxorrubicina.

Apesar de apresentar padrão semelhante ao da expressão de mRNA com HOX-1, o nível de proteína da HOX-2 foi elevado progressivamente juntamente com o aumento de concentração de doxorrubicina de $1 \mu \mathrm{M}$ a $5 \mu \mathrm{M}$, e baixou abruptamente abaixo do nível de linha de base quando 
tratado com $10 \mu \mathrm{M}$ de doxorrubicina. A diferença no perfil de alteração do nível de proteína entre HOX-1 e HOX2 pode ser devida à diferença em seus mecanismos de regulação. ${ }^{30,31} \mathrm{Em}$ ratos, a expressão de $\mathrm{HO}-2$ é modulada por glicocorticoides, por meio do elemento de resposta do glicocorticoide (GRE). ${ }^{32}$ Até onde sabemos, este é o primeiro estudo sobre o efeito da doxorrubicina na expressão de HOX-2 em cardiomiócitos. Entretanto, o mecanismo de ação preciso entre a doxorrubicina e a regulação de HOX-2 ainda é uma questão crítica não resolvida.

\section{Limitações do estudo}

Falta de resultados na detecção de atividades de enzimas em células H9c2 era considerado como a principal limitação de nosso estudo. Enquanto isso, o nível de stress oxidativo, de acordo com a alteração do metabolismo de heme não foi explorada, o que pode elucidar melhor a relação entre enzimas metabólicas do heme e cardiotoxicidade induzida por doxorrubicina. Mais estudos cobrindo esses aspectos são necessários para esclarecer o efeito da doxorrubicina na biossíntese e no metabolismo do heme.

\section{Conclusões}

O aumento da expressão de ALAS1 pode ter um papel na elevação do nível do heme quando o cardiomiócito H9c2 for exposto à doxorrubicina. Embora as HOX tenham sido reguladas para cima em concentrações de doxorrubicina de moderada a alta, seus efeitos degradadores foram sufocados pela ativação da síntese do heme não controlada.

\section{Referências}

1. Barbar T, Mahmood SS, Liu JE. Cardiomyopathy Prevention in Cancer Patients. Cardiol Clin. 2019 Nov;37(4):441-7.

2. Cuomo A, Rodolico A, Galdieri A, Russo M, Campi G, Franco R, et al. Heart Failure and Cancer: Mechanisms of Old and New Cardiotoxic Drugs in Cancer Patients. Card Fail Rev. 2019 May 24;5(2):112-8.

3. Sansbury BE, DeMartino AM, Xie Z, Brooks AC, Brainard RE, Watson LJ,et al Metabolomic analysis of pressure-overloaded and infarcted mouse hearts. Circ Heart Fail. 2014;7:634-42.

4. Khechaduri A, Bayeva M, Chang HC, Ardehali H. Heme levels are increased in human failing hearts. J Am Coll Cardiol. 2013;61:1884-93.

5. Rivankar S .An overview of doxorubicin formulations in cancer therapy. J Cancer Res Ther. 2014 Oct-Dec;10(4):853-8.

6. Liu H, Wang H, Xiang D, Guo W. Pharmaceutical Measures to Prevent Doxorubicin-Induced Cardiotoxicity. Mini Rev Med Chem. 2017;17(1):44-50.

7. Cappetta D, De Angelis A, Sapio L, Prezioso L, Illiano M, Quaini F, Rossi F, et al. Oxidative Stress and Cellular Response to Doxorubicin: A Common Factor in the Complex Milieu of Anthracycline Cardiotoxicity. Oxid Med Cell Longev. 2017;2017:1521020.

8. Gao G, Li J, Zhang Y, Chang YZ. Cellular Iron Metabolism and Regulation. Adv Exp Med Biol. 2019;1173:21-32.

9. Kafina MD, Paw BH. Intracellular iron and heme trafficking and metabolism in developing erythroblasts. Metallomics. 2017;9(9):1193-203.

10. Postnikova GB, Shekhovtsova EA. Myoglobin: Oxygen Depot or Oxygen
Os mecanismos específicos para a perda de controle de feedback negativo na formação do heme em tratamento com doxorrubicina, e o papel possível das ALAS como alvo terapêutico contra a citotoxicidade por heme induzida por doxorrubicina deverá ser estudada em investigações futuras.

\section{Contribuição dos autores}

Concepção e desenho da pesquisa e Redação do manuscrito: Wang Z; Obtenção de dados e Análise estatística: Wang Z, Gao J, Teng H; Análise e interpretação dos dados e Revisão crítica do manuscrito quanto ao conteúdo intelectual importante: Wang Z, Peng J.

\section{Potencial conflito de interesses}

Declaro não haver conflito de interesses pertinentes.

\section{Fontes de financiamento}

O presente estudo foi financiado pela Beijing Municipal Natural Science Foundation (Grant no 7162089).

\section{Vinculação acadêmica}

Não há vinculação deste estudo a programas de pósgraduação.

\section{Aprovação ética e consentimento informado}

Este artigo não contém estudos com humanos ou animais realizados por nenhum dos autores.
Transporter to Mitochondria? ANovel Mechanism of Myoglobin Deoxygenation in Cells (review). Biochemistry (Mosc). 2018 Feb;83(2):168-83.

11. Yanatori I, Richardson DR, Toyokuni S, Kishi F. How iron is handled in the course of heme catabolism: Integration of heme oxygenase with intracellular iron transport mechanisms mediated by poly $(\mathrm{rC})$-binding protein-2. Arch Biochem Biophys. 2019 Sep 15;672:108071.

12. Bhoite-Solomon V1, Kessler-Icekson G, Shaklai N. Myocyte injury by hemin In Vitro Cell Dev Biol Anim. 1993;29A (8):636-42.

13. Nath KA, Balla J, Croatt AJ, Vercellotti GM. Heme protein-mediated renal injury: a protective role for 21-aminosteroids in vitro and in vivo. Kidney Int. 1995;47(2):592-602.

14. Goldstein L, Teng ZP, Zeserson E, Patel M, Regan RF. Hemin induces an iron-dependent, oxidative injury to human neuron-like cells. J Neurosci Res. 2003;73(1):113-21.

15. Merle NS, Grunenwald A, Rajaratnam H, Gnemmi V, Frimat M, Figueres $\mathrm{ML}$, et al. Roumenina LT1,2, Intravascular hemolysis activates complement via cell-free heme and heme-loaded microvesicles. JCI Insight. 2018 Jun 21;3(12). pii: 96910.

16. Camus SM, De Moraes JA, Bonnin P, Abbyad P, Le Jeune S, Lionnet F, et al. Circulating cell membrane microparticles transfer heme to endothelial cells and trigger vasoocclusions in sickle cell disease. Blood. 2015 Jun $11 ; 125(24): 3805-14$

17. van der Vorm LN, Paw BH. Studying disorders of vertebrate iron and heme metabolism using zebrafish. Methods Cell Biol. 2017;138:193-220. 
18. Brown BL, Kardon JR, Sauer RT, Baker TA. Structure of the Mitochondrial Aminolevulinic Acid Synthase, a Key Heme Biosynthetic Enzyme. Structure. 2018 Apr 3;26(4):580-9.e4

19. Chiabrando D, Mercurio S, Tolosano E. Heme and erythropoieis: more than a structural role. Haematologica. 2014 Jun; 99(6):973-83.

20. Lathrop JT, Timko MP. Regulation by heme of mitochondrial protein transport through a conserved amino acid motif. Science. 1993;259(5094):522-5.

21. Lane DJ, Merlot AM, Huang ML, Bae DH, Jansson PJ, Sahni S, et al. Cellular iron uptake, trafficking and metabolism: key molecules and mechanisms and their roles in disease. Biochim Biophys Acta Mol Cell Res. 2015;1853:1130-44.

22. Peoc'h K, Nicolas G, Schmitt C, Mirmiran A, Daher R, Lefebvre T, et al. Regulation and tissue-specific expression of $\delta$-aminolevulinic acid synthases in non-syndromic sideroblastic anemias and porphyrias. Mol Genet Metab. 2019 Jan 23. pii: S1096-7192(18)30632-2.

23. Podvinec M, Handschin C, Looser R, Meyer UA. Identification of the xenosensors regulating human 5-aminolevulinate synthase. Proc Natl Acad Sci U S A. 2004;101(24):9127-9132.

24. Zordoky BN, El-Kadi AO. Induction of several cytochrome P450 genes by doxorubicinorubicin in H9c2 cells. Vascul Pharmacol. 2008 ;49(4-6):166-72.

25. Hedrich WD, Hassan HE, Wang H. Insights into CYP2B6-mediated drugdrug interactions. Acta Pharm Sin B. 2016 Sep;6(5):413-25.
26. Muñoz-Sánchez J, Chánez-Cárdenas ME. A review on hemeoxygenase-2: focus on cellular protection and oxygen response. Oxid Med Cell Longev. 2014;2014:604981.

27. Zhu XF, Li W, Ma JY, Shao N, Zhang YJ, Liu RM, et al. Knockdown of heme oxygenase-1 promotes apoptosis and autophagy and enhances the cytotoxicity of doxorubicin in breast cancer cells. Oncol Lett. 2015 Nov; 10(5):2974-80.

28. Morse D, Choi AM. Heme oxygenase-1: the "emerging molecule" has arrived. Am J Respir Cell Mol Biol. 2002 Jul;27(1):8-16.

29. Kitamuro T, Takahashi K, Ogawa K, Udono-Fujimori R, Takeda K, Furuyama K, et al. Bach1 functions as a hypoxia-inducible repressor for the heme oxygenase-1b gene in human cells. J Biol Chem. 2003 Mar $14 ; 278(11): 9125-33$

30. Dunn LL, Midwinter RG, Ni J, Hamid HA, Parish CR, Stocker R. New insights into intracellular locations and functions of heme oxygenase-1. Antioxid Redox Signal. 2014;20:1723-42.

31. Intagliata S, Salerno L, Ciaffaglione V, Leonardi C, Fallica AN, Carota G, et al. Heme Oxygenase-2 ( $\mathrm{HO}-2)$ as a therapeutic target: Activators and inhibitors. Eur J Med Chem. 2019 Dec 1;183:111703.

32. Liu N, Wang X, McCoubrey WK, Maines MD. Developmentally regulated expression of two transcripts for heme oxygenase- 2 with a first exon unique to rat testis: control by corticosterone of the oxygenase protein expression. Gene. 2000 Jan 4;241(1):175-83. 